\title{
BRANGESIAN SPACES IN THE POLYDISK
}

\author{
DINESH SINGH
}

(Communicated by Paul S. Muhly)

\begin{abstract}
In this paper we extend to the polydisk $D^{2}$ a theorem of $\mathrm{L}$. de Branges which characterizes the class of all Hilbert spaces that are contractively contained in the classical Hardy space $H^{2}$ of the disk and which are invariant under the shift $S$ acting as an isometry. Our theorem characterizes Hilbert spaces which are vector subspaces of $H^{2}\left(D^{2}\right)$ and which are invariant under the operators of multiplication by the coordinate functions whose actions are isometric and which doubly commute. We do not use contractivity.
\end{abstract}

\section{INTRODUCTION}

In [2] L. de Branges has proved a theorem which not only extends Beurling's [1] famous shift-invariant subspace theorem but also its vector-valued generalizations due to Peter Lax [5] and Paul Halmos [3]. The theorem of de Branges (scalar version) replaces the shift-invariant subspaces of Beurling's theorem by a class of Hilbert spaces of analytic functions which are contractively contained in the Hardy space $H^{2}$ of the open unit disk $D$ and on which multiplication by the coordinate function $z$ is an isometry.

In this paper we give a two-variable version of de Branges's theorem on the space $H^{2}\left(D^{2}\right)$ of analytic functions on the bidisk $D^{2}$. In fact, we establish something more general by dropping the contractivity condition. This gives us a larger class of Hilbert spaces from which de Branges-type spaces can be obtained easily as a particular case. We shall also show that the necessary and sufficient condition for Beurling-type theorems to be valid in $D^{2}$ [6] follows as a simple consequence. However, as was kindly pointed out to us by the referee, this consequence is a straightforward deduction from the much more elementary and far earlier work of Radlow [7].

\section{TERMINOLOGY AND PRELIMINARY RESULTS}

Let $D$ be the open unit disk in the complex plane and $T$ be its boundary, i.e. the unit circle. Let $D^{2}$ and $T^{2}$ be the Cartesian products of $D$ and $T$ respectively. Let $m$ denote the normalized two-dimensional Lebesgue measure

Received by the editors June 26, 1989.

1980 Mathematics Subject Classification (1985 Revision). Primary 32A35, 47A15.

Key words and phrases. Polydisk, de Branges's theorem. 
on $T^{2}$, so that if $T^{2}=\left\{\left(e^{i \theta_{1}}, e^{i \theta_{2}}\right): 0 \leq e_{1} \leq 2 \pi, 0 \leq e_{2} \leq 2 \pi\right\}$, then $d m=d \theta_{1} d \theta_{2} / 4 \pi^{2}$. For $p>0$, let $L^{p}\left(T^{2}, d \theta_{1} d \theta_{2}\right)$ be the Lebesgue spaces on $T^{2}$ and let $H^{p}\left(D^{2}\right)$ denote the class of both variable analytic functions on $D^{2}$ such that

$$
\operatorname{Sup}_{0 \leq r \leq 1} \frac{1}{4 \pi^{2}} \int_{T^{2}}\left|f\left(r e^{i \theta_{1}}, r e^{i \theta_{2}}\right)\right|^{p} d \theta_{1} d \theta_{2}<\infty .
$$

By means of the Poisson kernel $P\left[\left(r_{1} e^{i \theta_{1}}, r_{2} e^{i \theta_{2}}\right)\right]=P_{r_{1}}\left(\theta_{1}-\theta_{2}\right) \cdot P_{r_{2}}\left(\theta_{1}-\theta_{2}\right)$ (where $P_{r}(\theta)=1-r^{2} /\left(1-2 r \cos \theta+r^{2}\right)$ ), one can identify $H^{p}\left(D^{2}\right)$ with a closed subspace of $L^{p}\left(T^{2}, d \theta_{1} d \theta_{2}\right)$. In particular, $H^{2}\left(D^{2}\right)$ is identified with the class of all $L^{2}\left(T^{2}, d \theta_{1} d \theta_{2}\right)$ functions $f\left(e^{i \theta_{1}}, e^{i \theta_{2}}\right)$ which have Fourier series of the type $\sum_{m=0}^{\infty} \sum_{n=0}^{\infty} a_{m n} e^{i m \theta_{1}} e^{i n \theta_{2}}$ such that $\|f\|_{2}=\left(\sum_{m=0}^{\infty} \sum_{n=0}^{\infty}\left|a_{m n}\right|^{2}\right)^{1 / 2}<\infty$. We denote this class by $H^{2}\left(T^{2}\right)$, which is a Hilbert space under the above norm. $H^{\infty}\left(T^{2}\right)$ stands for the class of all essentially bounded functions in $H^{2}\left(T^{2}\right)$, which is a Banach algebra under the essential supremum norm. For further information we refer to [8].

A bounded linear operator $S$ on a Hilbert space $H$ is said to be an isometry if $\|S x\|_{H}=\|x\|_{H}$ for all $x$ in $H$. Let $S_{1}$ and $S_{2}$ be the operators on $H^{2}\left(T^{2}\right)$ given by

$$
S_{k} f\left(e^{i \theta_{1}}, e^{i \theta_{2}}\right)=e^{i \theta_{k}} f\left(e^{i \theta_{1}}, e^{i \theta_{2}}\right) \quad(k=1,2) .
$$

Obviously $S_{1}$ and $S_{2}$ are isometries on $H^{2}\left(T^{2}\right)$. It is also easy to see that $S_{1}$ commutes with $S_{2}^{*}$ (the adjoint of $S_{2}$ ) on $H^{2}\left(T^{2}\right)$. Two commuting operators $A, B$ on a Hilbert space $H$ are said to be doubly commuting if $A$ commutes with $B^{*}$, or equivalently if $B$ commutes with $A^{*}$. An isometry $S$ on $H$ is said to be a shift if $\bigcap_{n=0}^{\infty} S^{n}(H)=\{0\}$.

Let $G$ be a semigroup and let $S(t), t \in G$, be an abelian semigroup of isometries on a Hilbert space $H$. A closed subspace $L$ of $H$ is said to be a wandering subspace for $S(t)$ if $S\left(t_{1}\right) L \perp S\left(t_{2}\right) L$ for every $t_{1}, t_{2}$ in $G$, $t_{1} \neq t_{2}$. We shall need the following results from [12].

Lemma 2.1. Let $V_{1}, V_{2}$ be two doubly commuting isometries on a Hilbert space $H$ which are shifts. Then

(i) The semigroup $\left\{V_{1}^{m} V_{2}^{n}\right\}_{m, n \geq 0}$ is of type 's' (see [12, p. 255]) and

(ii) $L_{1} \cap L_{2}$ is a wandering subspace for the semigroup $\left\{V_{1}^{m} V_{2}^{n}\right\}_{m, n \geq 0}$ and $H=\sum_{m=0}^{\infty} \sum_{n=0}^{\infty} \oplus V_{1}^{m} V_{2}^{n}\left(L_{1} \cap L_{2}\right)$, where $L_{k}=H \theta V_{k}(H) \quad(k=1,2)$.

Proof. See [12, Theorem 1].

Lemma 2.2. Let $V_{1}, V_{2}$ be commuting isometries on the Hilbert space $H$. If $L_{1} \cap L_{2}=0$, where $L_{k}=H \theta V_{k} H$, then the semigroup $\left\{V_{1}^{m} V_{2}^{n}\right\} \quad m, n \geq 0$ is not of the type 's'.

Proof. See [12, Corollary 1]. 
A Hilbert space $H$ is said to be contractively contained in a Hilbert space $K$ if:

(i) $H$ is a vector subspace of $K$ and

(ii) $\|x\|_{K} \leq\|x\|_{H}$ for each $x$ in $H$.

\section{THE THEOREM OF DE BRANGES ON THE UNIT CIRCLE}

Let $g$ be any function in the unit ball of $H^{\infty}(T)$ and let

$$
M(g)=\left\{g\left(e^{i \theta}\right) f\left(e^{i \theta}\right): f\left(e^{i \theta}\right) \in H^{2}(T)\right\} .
$$

(For details on these classical $H^{p}(T)$ spaces, see [4] or [10]). $M(g)$ becomes a Hilbert space under the norm $\|g f\|_{M}=\|f\|_{2}$. It is easy to verify that $M(g)$ is contractively contained in $H^{2}(T)$ and that the shift operator, i.e. the operator of multiplication by the coordinate function $e^{i \theta}$, acts as an isometry on $M(g)$, which is invariant under the shift. The theorem of de Branges [9] says the converse:

Theorem 3.1. Let $M$ be a Hilbert space contractively contained in $H^{2}$ such that $M$ is invariant under the shift, which acts as an isometry on $M$. Then there is a unique $g$ in the unit ball of $H^{\infty}(T)$ (unique up to a factor of modulus one) such that $M=M(g)$.

In [11] we have observed that dropping the contractivity condition enables us to prove the more general result below. Let $g$ be any element of $H^{\infty}$ and let $N(g)=\left\{g f: f \in H^{2}(T)\right\} . N(g)$ is a Hilbert space under the norm $\|g f\|_{N}=\|f\|_{2}$. It is easy to see that $N(g)$ is contractively contained in $H^{2}$ if and only if $g$ is in the unit ball of $H^{\infty}$. It is also obvious that multiplication by the coordinate function $e^{i \theta}$ leaves $N(g)$ invariant and that it is an isometry on $N(g)$. The following is its converse.

Theorem 3.2. Let $N$ be a Hilbert space which is a vector subspace of $H^{2}(T)$ and such that multiplication by the coordinate function $e^{i \theta}$ leaves $N$ invariant and is an isometry on $N$. Then there exists a $g$ in $H^{\infty}$ (unique up to a factor of modulus one) such that $N=N(g)$.

Proof. See [11].

\section{THE MAIN RESULT}

Let $g\left(e^{i \theta_{1}}, e^{i \theta_{2}}\right)$ be in $H^{\infty}\left(T^{2}\right)$ and let

$$
N\left(g\left(e^{i \theta_{1}}, e^{i \theta_{2}}\right)\right)=\left\{g\left(e^{i \theta_{1}}, e^{i \theta_{2}}\right) f\left(e^{i \theta_{1}}, e^{i \theta_{2}}\right): f \in H^{2}\left(T^{2}\right)\right\} .
$$

$N(g)$ becomes a Hilbert space under the norm $\|g f\|_{N}=\|f\|_{2}$ where $\|f\|_{2}=$ $\left(\sum_{m=0}^{\infty} \sum_{n=0}^{\infty}\left|a_{m n}\right|^{2}\right)^{1 / 2}$ for $f\left(e^{i \theta_{1}}, e^{i \theta_{2}}\right)=\sum_{m=0}^{\infty} \sum_{n=0}^{\infty} a_{m n} e^{i m \theta_{1}} e^{i n \theta_{2}}$. It is also easy to verify that $S_{k}(N(g)) \subset N(g)(k=1,2)$, where $S_{k} f=e^{i \theta_{k}} f\left(e^{i \theta_{1}}, e^{i \theta_{2}}\right)$ and that $S_{1}, S_{2}$ are doubly commuting isometries on $N(g)$. The converse is our main result. 
Theorem. Let $N$ be a Hilbert space which is a vector subspace of $H^{2}\left(T^{2}\right)$ such that $S_{k}(N) \subset N(k=1,2)$ and $S_{1}, S_{2}$ are doubly commuting isometries on $N$. Then there exists a $g$ in $H^{\infty}\left(T^{2}\right)$ unique up to a factor of modulus one such that $N=N(g)$.

We first derive two consequences.

Corollary 1. Let $N$ be as in the above theorem and let it be contractively contained in $H^{2}\left(T^{2}\right)$. Then $g$ lies in the unit ball of $H^{\infty}\left(T^{2}\right)$.

Proof. Since $N=N(g)$ is contractively contained in $H^{2}\left(T^{2}\right)$, we have

$$
\begin{aligned}
\|g f\|_{2} & \leq\|g f\|_{N} \\
& =\|f\|_{2} \quad\left(\because\|g f\|_{N}=\|f\|_{2}\right) .
\end{aligned}
$$

Hence the norm of the operator of multiplication by $g$ is less than or equal to one. But this is easily seen to be the $H^{\infty}\left(T^{2}\right)$ norm of $g$. Hence $g$ lies in the unit ball of $H^{\infty}\left(T^{2}\right)$.

Corollary 2 [6, Theorem 2]. Let $N$ be a closed subspace of $H^{2}\left(T^{2}\right)$ such that $N$ is invariant under $S_{1}, S_{2}$, which doubly commute and acts as isometries on $N$. Then there is an inner function $q$ (unique up to a factor of modulus one) such that $N=q H^{2}\left(T^{2}\right)$.

Proof. It is obvious by the theorem that $N=N(q)=q H^{2}\left(T^{2}\right)$ for a unique $q$ in the unit ball of $H^{\infty}\left(T^{2}\right)$. Further, because $N$ has the same norm as that of $H^{2}\left(T^{2}\right)$, it follows that multiplication by $q\left(e^{i \theta_{1}}, e^{i \theta_{2}}\right)$ is an isometry on $H^{2}\left(T^{2}\right)$. Thus $\left|q\left(e^{i \theta_{1}}, e^{i \theta_{2}}\right)\right|=1$ a.e., i.e. $q$ is an inner function.

Remark. As was pointed out to us by the referee, Theorem 2 of [6] is an easy consequence of the much more elementary and far earlier work of Radlow [7].

Proof of the theorem. It is easy to see that $\bigcap_{n=0}^{\infty} S_{k}^{n}(N)=\{0\} \quad(k=1,2)$. Hence $S_{1}$ and $S_{2}$ are shifts on $N$. Setting $L_{k}=N \theta S_{k}(N) \quad(k=1,2)$, we get by Lemma 2.1 that $L_{1} \cap L_{2}$ is a wandering subspace for the semigroup $\left\{S_{1}^{m} S_{2}^{n}\right\}$ $m, n \geq 0$, and by Lemma 2.2 that $L_{1} \cap L_{2} \neq\{0\}$. Hence

$$
N=\sum_{m=0}^{\infty} \sum_{n=0}^{\infty} \oplus S_{1}^{m} S_{2}^{n}\left(L_{1} \cap L_{2}\right) \text {. }
$$

Let us choose a nonzero $g$ in $L_{1} \cap L_{2}$ such that $\|g\|_{N}=1$. Then $\left\{e^{i m \theta_{1}} e^{i n \theta_{2}} g\right\}$ $m, n \geq 0$ is an orthonormal sequence in $N$. Let

$$
f\left(e^{i \theta_{1}}, e^{i \theta_{2}}\right)=\sum_{m=0}^{\infty} \sum_{n=0}^{\infty} a_{m n} e^{i m \theta_{1}} e^{i n \theta_{2}}
$$

be any element of $H^{2}\left(T^{2}\right)$ and put

$$
f_{m n}=\sum_{k=0}^{m} \sum_{l=0}^{n} a_{k l} e^{i k \theta_{1}} e^{i l \theta_{2}}
$$


Then it is clear that $\left\{f_{m n}\right\}$ converges to $f$ in $H^{2}\left(T^{2}\right)$ as $m$ and $n$ tend to infinity by rectangles to be particular. Thus $\left\{f_{m n}\right\}_{m, n \geq 0}$ is a Cauchy sequence in $H^{2}\left(T^{2}\right)$. Now

$$
\begin{aligned}
\left\|f_{m n}\right\|_{2} & =\left(\sum_{k=0}^{m} \sum_{l=0}^{n}\left|a_{k l}\right|^{2}\right)^{1 / 2} \\
& =\left(\sum_{k=0}^{m} \sum_{l=0}^{n}\left\|a_{k l} g \cdot e^{i k \theta_{1}} e^{i l \theta_{2}}\right\|_{N}^{2}\right)^{1 / 2} \\
& =\left\|\sum_{k=0}^{m} \sum_{l=0}^{n}\left(a_{k l} g \cdot e^{i k \theta_{1}} e^{i l \theta_{2}}\right)\right\|_{N} \\
& =\left\|g \sum_{k=0}^{m} \sum_{l=0}^{n} a_{k l} e^{i k \theta_{1}} e^{i l \theta_{2}}\right\|_{N} \\
& =\left\|g f_{m n}\right\|_{N} .
\end{aligned}
$$

Hence $\left\{g f_{m n}\right\}_{m, n \geq 0}$ is a Cauchy sequence in $N$. As $N$ is a Hilbert space, it follows that there is an $h$ in $N$ such that $\left\{g f_{m n}\right\}$ converges to $h$ in $N$. Thus

$$
h=\sum_{m=0}^{\infty} \sum_{n=0}^{\infty} g \cdot a_{m n} e^{i m \theta_{1}} e^{i n \theta_{2}}
$$

so that letting $g=\sum_{m=0}^{\infty} \sum_{n=0}^{\infty} b_{m n} e^{i m \theta_{1}} e^{i n \theta_{2}}$, we have for any fixed positive $m$ and $n$,

$$
\begin{aligned}
h= & a_{00} g+a_{01} e^{i \theta_{2}} g+a_{10} e^{i \theta_{1}} g+\cdots+a_{m n} e^{i m \theta_{1}} e^{i n \theta_{2}} g \\
& +e^{i(m+1) \theta_{1}} h_{1}+e^{i(n+1) \theta_{2}} h_{2},
\end{aligned}
$$

where

$$
h_{1}=a_{0, n+1} g+a_{0, n+2} e^{i \theta_{2}} g+\cdots
$$

and

$$
h_{2}=a_{m+1,0} g+a_{m+2,0} e^{i \theta_{1}} g+\cdots .
$$

It is easily seen that $h_{1}$ and $h_{2}$ are in $N$ and hence in $H^{2}\left(T^{2}\right)$. Thus from the equation (2) above, we get that the $(m, n)$ th Fourier coefficient of $h$ is the same as $(m, n)$ th Fourier coefficient of the formal series, which is the formal product of the series of $g$ and $f$. This means that $g\left(e^{i \theta_{1}}, e^{i \theta_{2}}\right) \cdot f\left(e^{i \theta_{1}}, e^{i \theta_{2}}\right)$ is in $N$ and hence in $H^{2}\left(T^{2}\right)$. Further, $g f=h$. Since $\|h\|_{N}=\lim _{m, n}\left\|g f_{m n}\right\|_{N}=$ $\lim _{m, n}\left\|f_{m n}\right\|_{2}=\|f\|_{2}$, we conclude that for each $f$ in $H^{2}\left(T^{2}\right), g f$ is in $N$ and $\|g f\|_{N}=\|f\|_{2}$. We next show that $g$ is in $H^{\infty}\left(T^{2}\right)$ to establish $N(g) \subset N$. By the closed graph theorem, multiplication by $g$ is easily seen to be a bounded linear operator on $H^{2}\left(T^{2}\right)$, because $g f \in N \subset H^{2}\left(T^{2}\right)$ for 
each $f$ in $H^{2}\left(T^{2}\right)$. We can assume, without any loss of generality, that the norm of this operator is one. Let $t>1$ be arbitrarily chosen, and let $I=$ $\left\{\left(e^{i \theta_{1}}, e^{i \theta_{2}}\right):|g|>t\right\}$. Then for each integer $n>0$,

$$
\begin{aligned}
1 & \geq\left(\frac{1}{4 \pi^{2}} \int_{T^{2}}|g|^{2 n} \cdot 1 d \theta_{1} d \theta_{2}\right)^{1 / 2} \\
& \geq\left(\frac{1}{4 \pi^{2}} \int_{I}|g|^{2 n} \cdot 1 d \theta_{1} d \theta_{2}\right)^{1 / 2} \\
& \geq t^{n} \frac{1}{2 \pi} \int_{I} d \theta_{1} d \theta_{2} .
\end{aligned}
$$

As $t>1$, this can only happen when the Lebesgue measure of $I$ is zero. Thus $g$ is in $H^{\infty}\left(T^{2}\right)$.

We have thus far shown that $g H^{2} \subset N,\|g f\|_{N}=\|f\|_{2}$, and $g$ is in $H^{\infty}\left(T^{2}\right)$, i.e. $N(g) \subset N$. To complete the proof of the theorem, we must show that $N(g)=N$ and $g$ is unique up to a factor of modulus one.

We shall first establish that $N(g)=N$. To do this it is sufficient in view of the equality (1) to show that $L_{1} \cap L_{2}$ is one-dimensional. So suppose there is a $g_{1}$ in $L_{1} \cap L_{2}$ such that $g_{1} \perp g$. Then, by the same methods that were used for $g$ and in view of the equality (1) as well as the facts that $S_{1}$ and $S_{2}$ are isometries, we conclude that $N\left(g_{1}\right) \subset N$ and $N\left(g_{1}\right) \perp N(g)$. But then $g g_{1}$ is in $N\left(g_{1}\right)$ as well as $N(g)$. This forces $g g_{1}=0$ and hence $g_{1}=0$.

For the uniqueness, suppose there is a $g_{1}$ in $N$ such that $N=N(g)=$ $N\left(g_{1}\right)$. Then there are elements

$$
f=\sum_{m=0}^{\infty} \sum_{n=0}^{\infty} a_{m n} e^{i m \theta_{1}} e^{i n \theta_{2}} \text { and } h=\sum_{m=0}^{\infty} \sum_{n=0}^{\infty} b_{m n} e^{i m \theta_{1}} e^{i n \theta_{1}}
$$

such that $g=g_{1} h$ and $g_{1}=g f$. From this, we deduce that $\|g\|_{N}=1=$ $\left\|g_{1} h\right\|_{N}=\|h\|_{2}$. Similarly $\|f\|_{2}=1$. Further, we have $h f=1$. Hence from $\|h\|_{2}=1=\|f\|_{2}$ we conclude that $\left|a_{00}\right| \leq 1,\left|b_{00}\right| \leq 1$, and from $h f=1$ we conclude that $\left|a_{00} b_{00}\right|=1$. This means that $1=\left|a_{00}\right|=\left|b_{00}\right|$. So that from $\|h\|_{2}=1$, we get $h=a_{00}$ and $g=a_{00} g_{1}$. This completes the proof of the theorem as $\left|a_{00}\right|=1$.

\section{ACKNOWLEDGMENT}

The author is grateful to the referee for pointing out the work of Radlow [7] and its relevance.

\section{REFERENCES}

1. P. Beurling, On two problems concerning linear transformations in Hilbert space, Acta Math. 81 (1949), 239-255.

2. L. de Branges, Square summable power series (to appear).

3. P. R. Halmos, Shifts on Hilbert spaces, J. Reine Angew. Math. 208 (1961), 102-112. 
4. K. Hoffman, Banach spaces of analytic functions, Prentice-Hall, Englewood Cliffs, NJ, 1962.

5. P. Lax, Translation invariant spaces, Acta Math. 101 (1969), 163-178.

6. V. Mandrekar, The validity of Beurling theorems in polydisks, Proc. Amer. Math. Soc. 103, 145-148.

7. J. Radlow, Closed ideals in square summable power series, Proc. Amer. Math. Soc. 38 (1973), 293-297.

8. W. Rudin, Function theory in polydiscs, Benjamin, New York, 1969.

9. D. Sarason, Shift invariant spaces from the Brangesian viewpoint (Proc. Sympos. on the Occasion of the Proof of the Bierbach Conjecture, 1986), Math. Surveys Monogr., Amer. Math. Soc., Providence, RI, 1986, pp. 153-166.

10. __ Function theory on the unit circle, Virginia Polytech. Inst. and State Univ., Blacksburg, VA, 1979.

11. U. N. Singh and Dinesh Singh, An extension of a theorem of de Branges (to appear).

12. M. Slocinski, On the Wold type decomposition of a pair of commuting isometries, Ann. Polon. Math. 37 (1980), 255-262.

Department of Mathematics, University of Delhi, Delhi-1 10 007, India 